m28.p05

\section{Supermolecular Constitution of Natural Organic Minerals}

\section{Olga V. Kovaleva, Yevgeny A. Golubev}

Institute of Geology, Russian Academy of Sciences, 54, Pervomaiskaya st., 167982, Syktyvkar, Russia. E-mail: OlgaKovaleva@geo.komisc.ru

\section{Keywords: AFM, nanostructures, polymer structure and morphology}

Supermolecular constitution serves as a determing structural feature of many polymeric solid substances, particularly, such as natural organic minerals - solid hydrocarbons (bitumens). Basic supermolecular structural elements of solid bitumens are fibrils and globules. Their shape depends on correlation between energy of interaction of the macromolecules composing their shape and energy intra- and intermolecular interactions. The subject of our investigation is solid bitumens with a various degree of carbonization (asphaltites, kerites and anthraxolites) by atomic force microscopy (AFM).

Main regulations of supermolecular ordering of the solid bitumens are established. Asphaltites have a fibrillar composition and they locate randomly so that it is hard to determine a direction of the movement. The fiber diameter of them is about 200-400 nm. Less mutually oriented arrangement of the fibers are observed in the low-class kerites (the sizes of fibres is $200-300 \mathrm{~nm}$ ), the fibers become longer. The oriented arrangement of the fibers $(50-100 \mathrm{~nm})$ are preserved in the high-class kerites, however, the fibers become shorter, and globule-like formations (70-90 nm) are observed in the general fiber mass. When referring to the high-class anthraxolites globules $(10 \mathrm{~nm})$ represent the supermolecular structure, sometime the aggregate of globules $(30-50 \mathrm{~nm})$ are observed. The abstract is supported by Science School - 2250.2003.5.; Program \# 25.

\section{m28.p06}

\section{Gas Storage by Inclusion Compounds}

\author{
Tanya le Roex ${ }^{\mathrm{a}}$, Luigi Nassimbeni ${ }^{\mathrm{a}}$ \\ ${ }^{a}$ University of Cape Town, South Africa.E-mail:xraytan@science.uct.ac.za
}

\section{Keywords: supramolecular chemistry, gas sorption,} thermodynamics

Application of supramolecular chemistry and molecular recognition principles to the processes of gas storage, sensing and separation is developing rapidly. In this study, the absorption of gases by the host gossypol was investigated using apparatus designed to record gas sorption isotherms.[1] Gossypol is a yellow pigment found in cottonseed oil and is a symmetrically substituted 2,2'-binaphthalene. When crystallised from dichloromethane, gossypol forms three different inclusion compounds, one of which is a tubulate from which the dichloromethane guest molecules are easily removed, leaving behind the empty host framework [2] whose channels are thus available to absorb gas molecules. This gossypol clathrate was crystallised and desorbed and the empty host structure was found to absorb $\mathrm{CO}_{2}$. Experiments were carried out to measure the absorption or occupancy of $\mathrm{CO}_{2}$ at $30^{\circ} \mathrm{C}$ and at various pressures ranging from 1.7 bar to 31.4 bar. In addition, experiments to investigate the thermodynamics of this system were carried out, in which pressure was measured with change in temperature for various occupancies of $\mathrm{CO}_{2}$. Plots of $\mathrm{xln} P$ (where $\mathrm{x}$ is the occupancy or guest/host molar ratio) versus $1000 / \mathrm{T}$ were then used to calculate values of $\Delta \mathrm{H}$ at various occupancies.

[1] J.L. Atwood, L.J. Barbour, P.K. Thallapally and T.B. Wirsig, Chem. Commun., 2005, 51.

[2] B.T. Ibragimov, S.A. Talipov and T.F. Aripov, J. Inclusion Phenom., $17,1994,317$ 